\title{
High Spatial Resolution Analytical Electron Microscopic Investigation of Femtosecond-Laser-Induced Crystallization of a-Si:H Films
}

\author{
V.P. Oleshko*, B.K. Nayak ${ }^{* *}$ and M.C. Gupta ${ }^{* *}$ \\ *Department of Materials Science \& Engineering, University of Virginia, Charlottesville, VA \\ 22904-4745 \\ ** Department of Electrical \& Computer Engineering, University of Virginia, Charlottesville, VA \\ 22904-4743
}

Laser induced crystallization of thin amorphous semiconductor films is regarded as an attractive technique for fabrication of thin film transistors in active-matrix liquid-crystal displays [1,2] and of high-efficiency low-cost thin-film solar cells [3,4]. Typically nanosecond or microsecond lasers are utilized to crystallize such films via a rapid melting and solidification process. Ultra short laser pulse interaction with material introduces nonlinear photon energy absorption and nonequilibrium thermodynamics that are anticipated to govern the interactions [5-7]. Ultra short laser interactions with solid provide precise and low threshold fluence for material ablation [8-10]. It has been demonstrated that by controlling the laser fluence and number of pulses impinged on hydrogenated amorphous silicon thin films (a-Si:H), one can crystallize and texture the film at the same time [11]. This one step processing could have tremendous technological importance for fabrication of high efficient thin film solar cells and photo detectors. However, further in-depth investigation is necessary to understand the relations between structural changes, surface texturing and functional properties. This work is aimed to study the structural evolution of a-Si:H films after being exposed to a single femtosecond laser pulse using high spatial resolution field-emission analytical electron microscopy (FE-AEM) combining conventional and phase contrast electron imaging, selected-area electron diffraction (SAED) with energy-dispersive X-ray (EDX) and parallel electron energy-loss (PEEL) spectroscopies.

The samples for the study were a-Si:H single junction p-i-n type of solar cells fabricated on glass substrates using standard PECVD technique. The samples were placed on a $\mathrm{x}-\mathrm{y}$ stage and treated using a 120 femtosecond Ti : Sapphire laser system (Spectra Physics, Spitfire). The system delivered pulses at a repetition rate of $100 \mathrm{~Hz}$ and a wavelength of $800 \mathrm{~nm}$. The laser fluence was maintained at $0.24 \mathrm{~J} / \mathrm{cm}^{2}$. The beam spot on the sample was circular with beam diameter of $0.76 \mathrm{~mm}$. The samples were scanned in such a way that each point on the surface was exposed only to one laser shot. After that the specimen were collected on the microscopy grids. Bright-field and dark-field (BF-/DF-) TEM/ SAED/(HR) TEM, EDXS and PEELS of the films were done on a JEOL JEM$2010 \mathrm{~F}$ field-emission AEM operating at $197 \mathrm{kV}$ with a high-tilt pole piece $\left(\mathrm{C}_{\mathrm{s}}=1.0 \mathrm{~mm}\right.$ and $0.23 \mathrm{~nm}$ resolution by points) and utilizing a Gatan Imaging Filter, model 678 and an Oxford Pentafet ultrathin window EDX detector with resolution of $136 \mathrm{eV}(\mathrm{MnK} \alpha)$. PEEL spectra were acquired using a $0.6 \mathrm{~mm}$ entrance aperture in the image mode with $0.05-0.1 \mathrm{eV} /$ pixel dispersion and $1.0 \mathrm{eV}$ resolution at the zero-loss peak (FWHM). (HR)TEM/SAED revealed largely amorphous structure in the initial film (FIG. 1a). In the processed film, diamond type cubic Si crystalline sphere-like surface (and defect) structures and c-Si nanocrystals of different sizes and orientations grown in the amorphous matrix were found (FIGs. $1 \mathrm{~b}$ and 1c). Nanoprobe EDXS performed using nominal probe size varying from $2.4 \mathrm{~nm}$ to $0.5 \mathrm{~nm}$ indicated some surface oxidation of the film during laser processing. First derivatives of low-loss PEEL spectra (FIG. 2a) demonstrate a $0.4 \mathrm{eV}$ high-energy 
shift of the bulk plasmon at $16.7 \mathrm{eV}$ indicating an increase in density of the processed film. The net $\mathrm{SiL}_{2,3}$-edge (FIG. 2b) also shows a femtosecond-laser-induced low-energy shift of the ELNES features at $103.4 \mathrm{eV}$ and $111.6 \mathrm{eV}$ reflecting variations in the symmetry-projected density of $\mathrm{s}, \mathrm{p}$ and $\mathrm{d}$ conduction band states at $\Delta_{1}, \mathrm{~L}_{1}$ and $\mathrm{L}_{3}$ in the Brillouin zone.
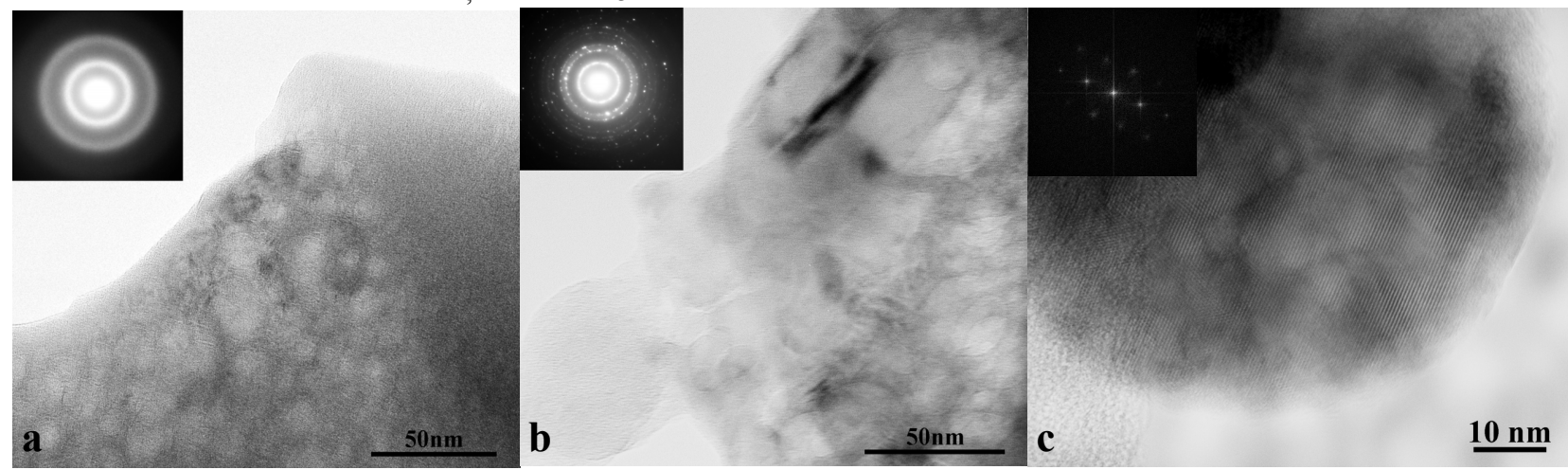

FIG. 1. TEM micrographs of (a) initial a-Si:H film and $(b, c)$ femtosecond laser-processed a-Si:H film. Insets in $(a, b)$ show corresponding SAED patterns. (c) HRTEM image and Fourier transform (inset) of a processed crystalline spherical particle demonstrating $\{111\}$ and $\{220\}$ lattice fringes.
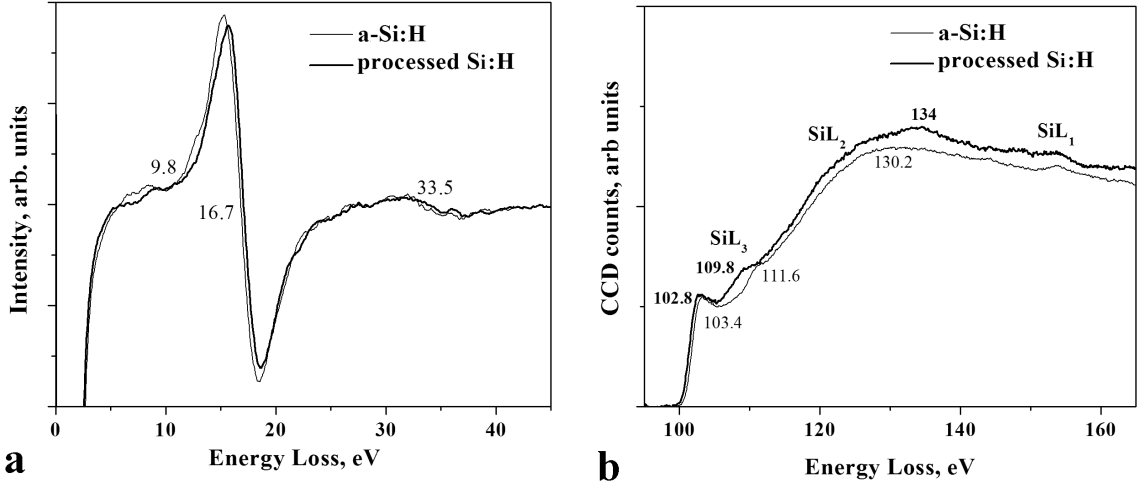

FIG. 2. PEEL spectra of untreated and femtosecond laser processed a-Si:H film. (a) First derivatives of low-loss PEEL spectra show the bulk plasmon $(16.7 \mathrm{eV})$, surface $(9.8 \mathrm{eV})$ and double $(33.5 \mathrm{eV})$ plasmons. (b) The net $\mathrm{SiL}_{2,3}$-edge.

\section{References}

[1] S.D. Brotherton, J.R. Ayres, M.J. Edwards, C.A. Fisher, C. Glaister, J.P. Gowers, D.J. McCulloch, M. Trainor, Thin Solid Films 337 (1999) 188. [2] M. Lee, S. Moon, M. Hatano, C. P. Grigoropoulos, Appl. Phys. 73 (2001) 317. [3] R.B. Bergmann, Appl. Phys. A 69 (1999) 187. [4] B.K. Nayak, B. Eaton, J.A.A. Selvan, J. Mcleskey, M.C. Gupta, R. Romero, G. Ganguly, Appl. Phys. A 80 (2005) 1077. [5] K. Sundaram, E. Mazur, Nat. Mater. 1 (2002) 217. [6] A. Rousse C. Rischel, S. Fourmaux, I. Uschmann, S. Sebban, G. Grillon, Ph. Balcou, E. Förster, J. P. Geindre, P. Audebert, J. C. Gauthier, D. Hulin, Nature 410 (2001) 65. [7] K. Sokolowski-Tinten, J. Biakowski, D. von der Linde, Phys. Rev. B 51 (1995) 14186. [8] T.Y. Choi, C.P. Grigoropoulos, J. Appl. Phys. 92 (2002) 4918. [9] X. Liu, D. Du, G. Mourou, IEEE J. Quantum Electron. 33 (1997) 1706. [10] T. Q. Jia Z. Z. Xu, X. X. Li, R. X. Li, B. Shuai, F. L. Zhao, Appl. Phys. Lett. 82 (2003) 4382. [11] B.K. Nayak, M.C. Gupta, Mater. Res. Soc. Symp. Proc. Vol. 850 (2005) MM1.8.1. [12] Authors acknowledge the support from the NSF, grant ECS-0100243 (BKN and MCG) and from the DOE, contract DE-FG02-01ER45918 (VPO). We also thank Dr. Yuan-Min Li of EPV Corp., NJ for providing a-Si:H films. 